\title{
Respective Answers to Coordinated Questions
}

\author{
Jean Mark Gawron and Andrew Kehler \\ San Diego State University and University of California, San Diego
}

\section{Introduction}

Munn $(1998,1999)$ observes that questions like (1a) can receive felicitous pair-list answers such as (1b) that appear to violate the identity condition on across-theboard (ATB) extraction:

(1) a. Where did Mary vacation and Bill decide to live?

b. Mary vacationed in Paris and Bill decided to live in Toronto.

That is, the ATB identity condition would require that Where denote the same location for the two gap sites in the conjoined predication.

However, Munn argues that such examples do not in fact violate the identity condition. He instead concludes that such cases should be analyzed as functional readings along the lines of Chierchia (1993), for whom pair-list readings are a variety of functional reading. The schematic semantics for (1a) in this analysis is as shown in (2):

(2) Which $f$ : Mary vacationed in $f($ Mary) and Bill decided to live in $f($ Bill $)$ ?

As such, what is being requested by (1a) in this view is a specification of a function through the enumeration of argument-value pairs, that is, the function that when applied to Mary gives the place she vacationed, and when applied to Bill gives the place he decided to live.

Munn follows Chierchia in implementing the analysis at LF, per the representation in (3).

[Where $]_{i}$ did Mary vacation $_{i}^{x}$ and Bill $_{y}$ decide $\mathrm{t}_{i}^{y}$ to live?

The key component of the analysis concerns the doubly-indexed "functional" traces. The subscript represents a function associated with the trace, and is bound by the wh-operator. The superscript is an argument index which is bound locally, in this 
case by the respective subjects of the two clauses. Since the same function is extracted from each clause, the identity condition is purportedly met.

However, there are a couple of anomalies that arise in this analysis. First, it specifically predicts that questions with a conjoined VP such as (4a) cannot have pair-list answers such as (4b), because in such cases only a single binder is available for the two traces (Bill in 4a). We find the answer in (4b) to be impeccable, however.

a. What did Bill eat and drink?

b. He ate a hamburger and drank a coke.

The remaining option for a functional analysis (which Munn does not pursue) is to take the verb denotations to be the function arguments, but this move would essentially require that traces be coindexed with verbs as in (5), which is no doubt an unappealing prospect.

$$
[\text { What }]_{i} \text { did Bill eat } \mathrm{t}_{i}^{x} \text { and } \operatorname{drink}_{y} \mathrm{t}_{i}^{y} \text { ? }
$$

Second, whereas Munn's non-identity ATB readings are available with whNPs that are unspecified for number (as in 1a \& 4a), they become unavailable with NPs that are unambiguously singular: ${ }^{1}$

a. In what city did Mary vacation and Bill decide to live?

b. \# Mary vacationed in Paris and Bill decided to live in Toronto. (but fine with cities instead of city in 6a)

This restriction is unexpected since it does not arise with other cases that have been successfully analyzed as having pair-list readings, such as the following variant of an example from Chierchia (1993):

a. What relative does every boy love?

b. Tom loves his mother, Fred loves his aunt...

In this paper, we argue that the pair-list readings of examples like (1a) are not functional, but are instead instances of respective readings of the sort operative in the variant shown in (8),

Mary vacationed and Bill decided to live in Paris and Toronto (respectively).

and provide an analysis using the semantic machinery for such readings proposed by Gawron and Kehler (in press). We then return to the set of data Munn considers and argue that it is not uniform; indeed we claim that one particular subclass of cases he considers are functional. 


\section{The Semantics of Respective Readings}

We start by summarizing the analysis of respective readings of Gawron and Kehler (in press). Several examples that provide adequacy criteria for any such analysis motivate our approach. A basic case of a respective reading is shown in (9a):
a. Tolstoy and Dostoyevsky wrote Anna Karenina and The Idiot respec- tively.
b. Tolstoy wrote Anna Karenina and Dostoyevsky wrote The Idiot.

While the syntactic structure of (9a) looks to be rather pedestrian, the semantic relations expressed, paraphrased in (9b), belie this structure. While it might be tempting to argue that the pairwise dependencies manifest in the semantics are also represented in the syntax, many examples demonstrate that this proposal is untenable. Example (10) is from Dalrymple and Kehler (1995):

(10) Though the Trail Blazers won this series in six games from Phoenix, they were far from dominant. Their margins of victory were 2 points, 1 point, 6 points, and 3 points respectively. (New York Times article)

Whereas two groups of four entities are being paired in this example, only one of the relevant NPs contains four conjuncts. The (nonconjoined) NP their margins of victory instead obtains its denotation through reference and inference: reference to the previously mentioned set of six games, and inference from these to the four that the Trail Blazers won. An ordering on these four games is also inferred, specifically the temporal order in which they were played. Dalrymple and Kehler found that $14 \%$ of the respectively constructions in their corpus were of this sort, that is, they involved a mapping over sets of entities denoted by phrases with unequal numbers of conjuncts.

Since the predication relationships in these constructions cannot be read directly off of the syntax, another possibility is that the mechanism for their recovery can be attributed specifically to the occurrence of the adverb respectively. This tack will not work either; passage (10), for example, has the same reading as its preferred interpretation even if respectively is omitted, as shown in (11).

(11) Though the Trail Blazers won this series in six games from Phoenix, they were far from dominant. Their margins of victory were 2 points, 1 point, 6 points, and 3 points.

The recovery of these relationships will therefore have to be addressed within the realm of the semantics of plural predication. Complicating this task is the fact that respective readings can involve groups of property-denoting expressions. Examples (12-15) demonstrate the point with respect to conjoined VPs, verbs, adverbs, and adjectives respectively: 
(12) Sue and Karen jog to school and drive to work (respectively).

(13) George and Martha respectively denounced and were denounced by the governor. (McCawley 1998)

(14) John and Mary sang loudly and softly (respectively).

(15) The Mondavi Woodbridge and Beringer Reserve wines are cheap and expensive (respectively).

We therefore need a representation for groups of property-denoting and propositiondenoting expressions from which the subparts are retrievable. (In general, when we say 'property' we also mean to include relations.) To do this, we treat groups of these expressions as denoting SUMS, following Link's (1983) well-known analysis of groups of individual-denoting expressions in his treatment of the semantics of collective predication. (Authors who have previously used a notion of proposition sum include Lahiri (2000) and Krifka (2001).) Following previous theories of coordination (Gazdar 1980, Partee and Rooth 1983, Winter 1996), this proposal provides a treatment for conjunction that is uniform for property-denoting, proposition-denoting, and individual-denoting expressions. Conjunction is represented uniformly as the JOIN operation, although we write it as $\sqcup$ for properties and propositions and as $\vee$ for individuals.

Details of the lattice-theoretic construction we assume, as well as our type system given the need to move away from classic Montagovian denotations, are provided in Gawron and Kehler (in press). Here we explain what it means to predicate a boolean sum of an individual, per the following reduction axiom:

BOOLEAN REDUCTION: For each boolean type $\langle\mathrm{a}, \mathrm{b}\rangle$ :

$$
\begin{aligned}
\forall \alpha \in \mathrm{D}_{\mathrm{a}}, & \beta_{1} \beta_{2} \ldots \beta_{n} \in \mathrm{D}_{\langle\mathrm{a}, \mathrm{b}\rangle} \\
& {\left[\left[\beta_{1} \sqcup \beta_{2} \sqcup \ldots \sqcup \beta_{n}\right](\alpha)=\beta_{1}(\alpha) \sqcup \beta_{2}(\alpha) \sqcup \ldots \sqcup \beta_{n}(\alpha)\right] }
\end{aligned}
$$

This axiom yields the sum that results from applying each member of a function sum to an argument. For instance, a sum of one-place properties applied to an individual will yield a proposition sum.

Now that we have property sums, we explain how they are obtained. There are essentially three ways. First, and most obviously, they arise from the conjunction of property (and relation) denoting expressions. As such, the meaning of the conjoined VPs in (16a) is the property sum shown in (16b).
a. jog and drive
b. jog $\sqcup$ drive 
Second, property sums can arise in cases of sentential conjunction in which lambda abstraction has applied. For instance, the representation of the conjoined relative clauses in (17a) is as shown in (17b). It can be shown that (17b) is a property sum. $^{2}$

a. (the person) that John fired and Susan hired

b. $\lambda s[\operatorname{fired}(\mathrm{J}, s) \sqcup \operatorname{hired}(\mathrm{S}, s)]$

Lastly, property sums can result from the application of a distributivity operator along the lines of that used by Link (1983) and Roberts (1987), reformulated as shown in (19a) to produce a property sum.

wrote Anna Karenina and War and Peace

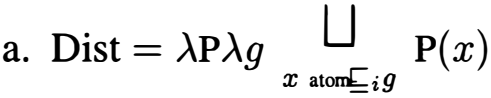
b. Dist(wrote) $=\lambda g \underset{x \text { atom }_{i} g}{\bigsqcup} \operatorname{wrote}(x)$

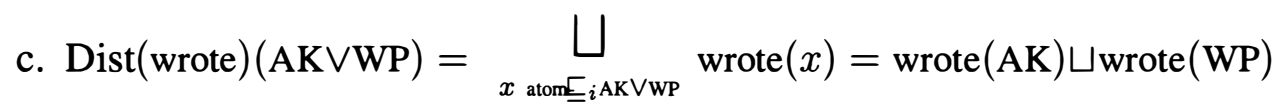

The distributivity operator (19a) applied to the transitive verb wrote in (18) yields (19b), which when applied to the individual sum denoting Anna Karenina and War and Peace yields the sum of propositions shown in (19c).

Now that we have property sums and ways to derive them, we now need a way to model the context-dependent ordering among members of sums. We do this with pragmatically-supplied SEQUENCING FUNCTIONS:

$$
f \in\left(\mathbf{U}^{\mathbf{I}}\right)^{\mathbf{G}}
$$

Here we use $\mathbf{U}$ for the set of (possibly atomic) entities of all types, $\mathbf{G}$ for the set of entities of all types minus atoms (i.e., the set of sums of all types), and $\mathbf{I}$ for the set of integers. A sequencing function $f$, then, is a partial function from $\mathbf{G}$ to an assignment function, where an assignment function is, in turn, a partial function from integers to entities.

Let us illustrate with example (21a):
a. Sue and Karen jog and drive respectively.
b. Sue, Karen, and Bob jog, drive, and jog respectively.
c. \# Sue, Karen, and Bob jog and drive respectively. 


\begin{tabular}{|c|c|c|}
\hline$i$ & \multicolumn{2}{|c|}{ Group } \\
\hline & $f(\mathbf{s} \vee \mathbf{k})(i)$ & $f($ jog $\sqcup$ drive $)(i)$ \\
\hline \hline 1 & $\mathbf{s}$ & jog \\
\hline 2 & $\mathbf{k}$ & drive \\
\hline 3 & $\uparrow$ & $\uparrow$ \\
\hline
\end{tabular}

Table 1: The sequencing function for example (21a), where $|f|=2$.

The sequencing function for (21a) is inferred from the order in which the members of each group are mentioned, as shown in Table 1. Thus, for index $i$ the sequencing function will return the $i$ th mentioned element in each group. The difference in felicity between sentences (21b) and (21c) is of interest because on most semantic theories, including ours, the two VPs have the same denotation. The difference is that the two mentions of jog in (21b) license its correspondence to two different indices of the inferred sequencing function as shown in Table 2, whereas the single mention of it in (21c) does not.

\begin{tabular}{|c|c|c|}
\hline$i$ & \multicolumn{2}{|c|}{ Group } \\
\hline & $f(\mathbf{s} \vee \mathbf{k} \vee \mathbf{b})(i)$ & $\begin{array}{l}f(\text { jog } \sqcup \text { drive } \sqcup \text { jog })(i)= \\
f(\text { jog } \sqcup \text { drive })(i)\end{array}$ \\
\hline \hline 1 & $\mathbf{s}$ & jog \\
\hline 2 & $\mathbf{k}$ & drive \\
\hline 3 & $\mathbf{b}$ & jog \\
\hline 4 & $\uparrow$ & $\uparrow$ \\
\hline
\end{tabular}

Table 2: The sequencing function for example (21b), where $|f|=3$.

Sequencing functions are subject to certain constraints, listed below:

1. Cardinality: We require that for all $g \in \mathbf{G}, f$ be defined for the same subset of $\mathbf{I}$. The cardinality of $f$ is written as $|f|$.

2. Proper Subgroups: We require that, for each $g, i, f(g)(i)$ pick out a proper subpart of $g$. That is,

$$
f(g)(i) \sqsubset_{i} g
$$

3. Exhaustivity: Finally, we require that $f(g)$ be exhaustive, that is, that:

$$
\left(\bigsqcup_{i \leq|f|} f(g)(i)\right)=g
$$

Additional details are provided in Gawron and Kehler (in press). It is worth noting here, however, that the subgroup and exhaustivity constraints conspire to require that sequencing functions have a cardinality of at least two, and thus can only be used by operators that are applied to plural denotations. 
In the discussion period after the presentation of this paper, we were asked about whether orderings among members of group-denoting NPs should be represented in the denotations themselves (i.e., as sequences), rather than modeled pragmatically via sequencing functions. Two manifestations of such an approach come to mind; we believe both are inferior to the present account.

First, group-denoting NPs could be treated as ambiguous between sequenced denotations, as would be required for the RESP operator, and ones without orderings, as would be required for other operators such as DIST. Example (24) illustrates why this option is untenable.

(24) Exercising their right under Utah law, a serial killer, Roberto Arguelles, and Troy Michael Kell, a white supremacist who stabbed a fellow inmate to death, have chosen the firing squad over lethal injection and are set to die at 12:01 a.m. on June 27 and 28, respectively. ("Utah prepares for 2 firingsquad executions in June", CNN website, 5/22/03)

In this example, a single group-denoting subject NP is coupled with a conjoined VP in which the first VP requires a distributive interpretation and the second requires a respective interpretation (as forced by the occurrence of respectively). An analysis that requires different types of subject NP denotation for these two readings predicts that such examples are unacceptable, since the subject NP cannot simultaneously have both interpretations. This example is reminiscent of previously discussed cases that show that distributive/collective ambiguities need to be located in the predicate rather than subject NP (Massey 1976, Schwarzschild 1996), such as (25):

John and Mary met and ate a hamburger.

To get the reading on which John and Mary each ate different hamburgers, a distributive reading is required for the second predicate and a collective reading is required for the first, which again is problematic if the subject NP can only have one of these interpretations. The same reasoning applies for the distributive/respective reading of the (naturally-occurring) example (24).

A second possible analysis would specify that all group denotations be represented only as sequences. This option comes at the cost of requiring that sequence information be computed and propagated throughout the semantic composition for all sentences that involve group denotations, even though almost all semantic operators will ignore this information. More importantly, this approach would appear to abandon any effort to factor apart those aspects of the interpretation that are determined by pragmatics - including the orderings of groups, as shown by examples like (10) - and those aspects that are determined by syntax. Sequencing functions provide this factorization, and also avoid the need to encode sequence information for all the different ways in which a group can be expressed in the grammar (e.g., conjoined phrases, plural pronouns and other referential NPs, and combinations 
thereof). Indeed, Schwarszchild (1996) argues persuasively for a pragmaticallydriven account of both respective readings and related examples of INTERMEDIATE DISTRIBUTIVITY such as (26):

The cows and the pigs filled the barn to capacity.

His account utilizes the notion of a pragmatically-determined COVER that partitions a group into a set of subgroups, which is capable of deriving the reading of (26) in which the cows together filled the barn to capacity and the pigs together filled the barn to capacity. It turns out that sequencing functions are a generalization of covers; see Gawron and Kehler (in press) for further discussion.

We are now ready to define the respective operator (RESP), shown in (27):

$$
\operatorname{Resp}_{f}=\lambda \mathbf{P} \lambda \mathrm{g} \underset{i \leq|f|}{\bigsqcup}[f(\mathrm{P})(i)](f(\mathrm{~g})(i))
$$

The RESP operator simply takes two sums and applies the $i$ th member of the first to the $i$ th member of the second, yielding a sum of the results for all $i$.

Derivation (28) shows the application of the operator to the sums containing the denotations for jog and drive and Sue and Karen in example (21a), which gives the desired proposition sum.

$$
\begin{aligned}
& \operatorname{Resp}_{f^{2}}(\text { jog } \sqcup \text { drive })(\mathbf{s} \vee \mathbf{k}) \\
& =\bigsqcup_{i \leq 2}[f(\text { jog } \sqcup \text { drive })(i)](f(\mathbf{s} \vee \mathbf{k})(i)) \\
& =\operatorname{jog}(\mathbf{s}) \sqcup \operatorname{drive}(\mathbf{k})
\end{aligned}
$$

This operator serves as the denotation of respectively in our analysis. Thus, the appearance of respectively in a sentence will force the introduction of a RESP operator into the semantic composition. However, like the DIST operator shown in (19a), the RESP operator can also occur freely, which is necessitated by examples such as (11).

The analysis of our basic case example (9a), repeated in (29), utilizes a freely-occurring DIST operator. The syntactic structure for (29) in shown in Figure $1 .{ }^{3}$ The semantic composition proceeds as before; the denotations of the nodes of the tree in (1) are shown in (30).

(29) Tolstoy and Dostoyevsky wrote Anna Karenina and The Idiot respectively.

a. 【[ Dist wrote ] Anna K and The Idiot 》

$$
\begin{aligned}
& =\operatorname{Dist} \text { write }(\mathrm{AK} \vee \mathrm{I}) \\
& =\text { write }(\mathrm{AK}) \sqcup \text { write }(\mathrm{I})
\end{aligned}
$$

b. 【 [ Dist wrote ] Anna $\mathrm{K}$ and The Idiot respectively 》

$$
=\operatorname{Resp}_{f^{2}}[\text { write }(\mathrm{AK}) \sqcup \text { write }(\mathrm{I})]
$$


c. [ Tolstoy and Dostoyevsky [ Dist wrote ]

Anna $\mathrm{K}$ and The Idiot respectively

$=\operatorname{Resp}_{f^{2}}[$ write $(\mathrm{AK}) \sqcup$ write $(\mathrm{I})](\mathrm{T} \vee \mathrm{D})$

$=\operatorname{write}(\mathrm{AK})(\mathrm{T}) \sqcup$ write $(\mathrm{I})(\mathrm{D})$

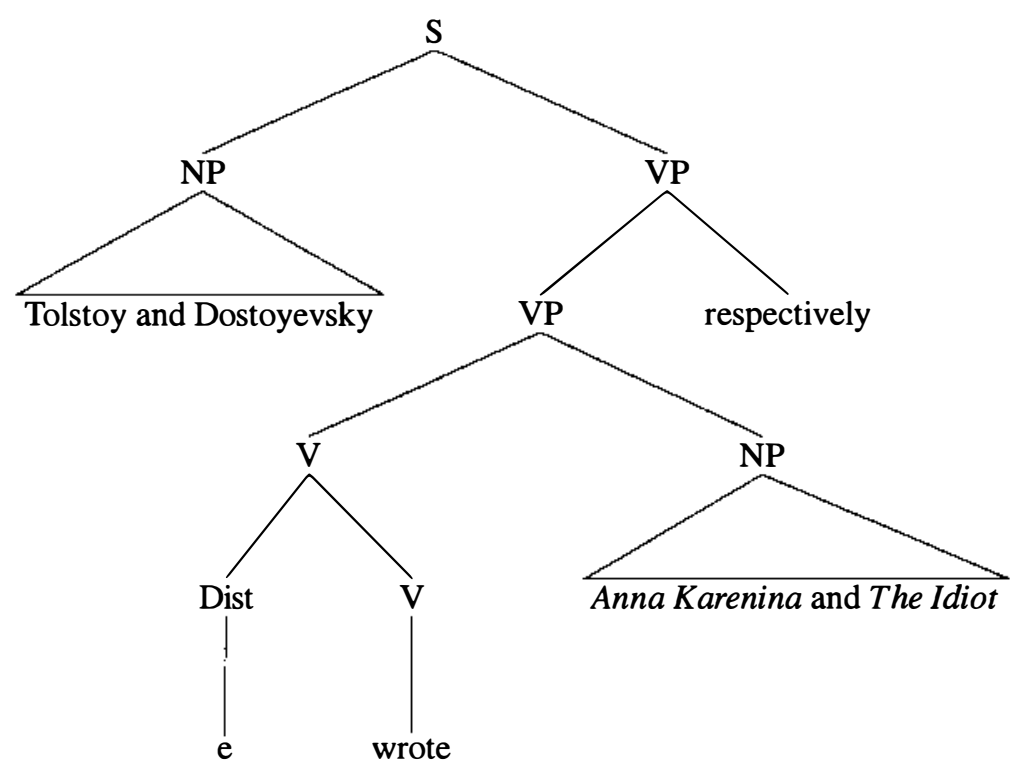

Figure 1: Analysis of (29)

The intervention of the DIST operator during the semantic composition (applying to the denotation of wrote) is responsible for the RESP operator ultimately receiving a property sum as an argument.

\section{Interaction will Filler-Gap Dependencies}

In the analysis just presented, respective readings occur when the RESP operator intervenes between constituent-based dependencies in the syntax and predicateargument dependencies in the semantics. We now consider what happens when a RESP operator comes into the semantic analysis of a filler-gap construction. Recall from our discussion of example (17) that conjunctions of gap-containing clauses naturally yield property sums:

a. (the person) that John fired and Susan hired (=17)

b. $\lambda s[\operatorname{fired}(\mathrm{J}, s) \sqcup \operatorname{hired}(\mathrm{S}, s)]$

As such, there is nothing in the analysis to stop the RESP operator from intervening between a sum of gap-containing clause denotations and a sum representing the denotations of the gap fillers, even though the result of applying the operator will 
generate a reading that violates the identity condition on ATB extraction. This is in fact the desired behavior; consider the case of conjoined relative clauses in (32).

(32) I bought travel guides for Paris and London yesterday. They are the cities where Mary vacationed and Bill decided to live, respectively.

The ATB reading would have Mary vacationing and Bill deciding to live in the same (two) cities, whereas the actual interpretation distributes one city to each predication. Although such examples counterexemplify any syntactic analysis that requires gaps to be coindexed with their fillers, these readings are expected on our account. The schematic semantics for (32) is shown in (33), in which the RESP operator intervenes between the sum of relative clause denotations and the sum representing the gap fillers.

$$
\text { the } c \operatorname{cities}(c)\left[\operatorname{Resp}_{f}(\lambda x[\operatorname{vacation}(\mathbf{M}, x) \sqcup \operatorname{decide-to-live-in}(\mathrm{B}, x)])(c)\right]
$$

It is worth noting that an analysis of (32) as involving a "lazy" pronominal reference - in which the conjoined NP Paris and London is syntactically copied to the site of the pronoun they in the second sentence - will not ultimately suffice, as can be seen by considering example (34):

(34) The two most expensive cities in Europe, which are where Mary vacationed and Bill decided to live respectively, are very beautiful.

In this case, the gap fillers are not even individuated in the context; their ordering is inferred from the semantics of the subject NP itself. Since RESP is freely-occurring, these analyses are available even if the adverb respectively does not appear.

Examples (35-40) show pairs similar to (32) and (34) for a range of other filler-gap constructions - including clefts (35-36), right-node raising (37-38), and topicalization (39-40) - all of which plausibly involve property extraction and thus introduce property sums as an argument to the RESP operator.

(35) I bought travel guides for Paris and London yesterday. It was these cities where Mary vacationed and Bill decided to live, respectively.

(36) It was the two most expensive cities in Europe where Mary vacationed and Bill decided to live, respectively.

(37) I bought travel guides for Paris and London yesterday. Mary vacationed, and Bill decided to live, in these two cities respectively.

(38) Mary vacationed, and Bill decided to live, in the two most expensive cities in Europe, respectively. 
(39) I bought travel guides for Paris and London yesterday. Those two cities, Mary vacationed in and Bill decided to live in, respectively.

(40) The two most expensive cities in Europe, Mary vacationed in and Bill decided to live in, respectively.

While there is clearly a syntactic dependency involved in such constructions, an identity constraint - which happens to be maintainable when a distributive reading is operative, for example - is too strong of a restriction in the general case, and thus should neither be predicted by nor stipulated within a syntactic theory of longdistance dependencies.

\section{Coordinated Questions}

In light of the examples discussed in the previous section, Munn's examples of coordinated questions can now be seen to be just another type of filler-gap construction in which a respective operator may intervene during the semantic composition. Consider again example (41a), with possible answers (41b) and (41c).
a. Where did Mary vacation and Bill decide to live? (=1a)
b. Paris and London.
c. Mary vacationed in Paris and Bill decided to live in London.

The schematic semantics for (41a) is shown in (42).

$$
\text { which } \mathbf{X} \operatorname{Resp}_{f}(\lambda \mathbf{x}[\operatorname{vacation}(\mathbf{M})(x)] \sqcap \lambda \mathbf{x}[\text { decide-to-live-in }(\mathrm{B})(x)])(\mathrm{X})
$$

This semantics has essentially the same form as that of the relative clause example (32) shown in (33), except that here the RESP operator intervenes between the semantics of the question and the answer. ${ }^{4}$ Thus, instead of viewing such questions as a request for a function specification per Munn's analysis, on our view such questions request a sum with an inferrable ordering on its members.

This analysis does not suffer from the drawbacks of Munn's account that were discussed in Section 1. First, the fact that sequencing functions are only defined for sums of cardinality greater than or equal to two predicts that the respective interpretation is unavailable with unambiguously singular NPs:
a. In what city did Mary vacation and Bill decide to live?
b. \# Paris and London (respectively).
c. \# Mary vacationed in Paris and Bill decided to live in London. 
Of course, both responses are fine if the question contains a plural NP, which is again as predicted:

a. In what cities did Mary vacation and Bill decide to live?

b. Paris and London (respectively).

c. Mary vacationed in Paris and Bill decided to live in London.

Second, recall that pair-list readings for cases involving VP conjunction, as in (45a), were predicted to be impossible on Munn's analysis. There is no such restriction on respective readings, hence the coherent schematic semantics that the analysis derives for (45a) shown in (45b).
a. What did Bill eat and drink? (=4a)
b. what $\mathrm{X} \mathrm{Resp} f(\lambda \mathbf{x}[\operatorname{eat}(\mathrm{B})(\mathrm{x})] \sqcap \lambda \mathrm{x}[\operatorname{drink}(\mathrm{B})(\mathrm{x})])(\mathrm{X})$

The same point applies to cases involving NP conjunction. For instance, the question in (46a) can felicitously receive a pair-list answer of the form in (46b).

a. What cities did Mary visit in Africa and Asia?

b. She visited Nairobi and Monrovia in Africa and Tokyo and Bangkok in Asia.

This fact is again captured on our analysis, but is problematic for Munn's, since Africa and Asia are configurationally unsuitable to serve as the binders necessary to license a functional reading.

An anonymous reviewer (David Beaver) points out that respective interpretations of coordinated questions involving property sums do not always seem to be as accessible as other possible readings if the adverb respectively does not occur, as in (47).

Which men and women do you like and love?

We agree with him on this point, but do not find the respective reading to be impossible for such examples, which is consistent with the fact that (47) is felicitous when respectively is included:

(48) Which men and women do you like and love respectively?

In light of this and the existence of respective readings for examples without respectively like (11), we believe that the most parsimonious account is one which also predicts the reading to be available in (47), even if dispreferred. The role of respectively in (48) is therefore to override what would otherwise be the preferred interpretation by explicitly introducing a RESP operator. Support for this claim is provided by the fact that other types of contextual clues can also help make the respective reading preferred. Consider example (49): 
(49) A. Let's gossip. Which man does Bob like the most?

B. Fred.

A. Which woman does he love the most?

B. Sue.

A. OK, which man and woman does John like and love the most?

B. Bob and Wanda.

The separate pairing made in the first four turns between men and who Bob likes on the one hand, and women and who he loves on the other, appears to make the respective interpretation of the question in the fifth turn preferred. Since, in a sense, respective readings take a certain amount of 'work' to compute - requiring the identification and application of a sequencing function - it is perhaps not surprising that additional contextual support or an overt use of respectively may be necessary to make such readings preferred.

\section{Another Look at Munn's Data}

We now step back and reanalyze the set of data that Munn discusses and the judgments that both he and we assign to them. We argue that in fact his examples are not uniform with respect to the analysis they should receive, and in particular, that one subclass of cases he considers are in fact instances of functional readings.

Let us begin by acknowledging some discrepancies between his and our judgments. We and Munn agree that cases involving number-ambiguous wh-NPs such as (50) are acceptable, although for different reasons.

a. Who did Bill kill on Tuesday and Fred kill on Wednesday?

b. John killed Bruno and Bill killed Arno.

The wh-NP Who is unspecified for number. Munn's analysis predicts that the pairlist response in (50b) is acceptable insofar as Who is singular, whereas we make this prediction insofar as it is plural. On the other hand, we disagree about the acceptability of cases in which the number of the NP is specified. That is, Munn finds the pair-list answer to (51a) shown in (51b) to be acceptable, whereas we find it to be deviant. On the other hand, we find the response impeccable if man in (51a) is replaced with the plural men. ${ }^{5}$

a. What man did Bill kill on Tuesday and Fred kill on Wednesday? (vs. men)

b. John killed Bruno and Bill killed Arno. (Munn's judgment)

Although he claims that (51) is acceptable, he nonetheless states that "the data improve considerably if we explicitly mention the function in the $w h$-phrase itself", citing example (52). 
(52) a. Which of his victims did John kill on Tuesday and Bill kill on Wednesday?

b. John killed Bruno and Bill killed Amo.

We agree with his judgment of (52), and find a marked contrast between it and (51).

The wh-phrase in example (52a) contains an embedded plural NP which is likely to prove irrelevant to the point at hand. So let us instead consider a variant in which the NP singular, shown in (53a).

a. Which victim on his list did John kill on Tuesday and Bill kill on Wednesday?

b. John killed Bruno and Bill killed Arno.

c. His brother. (with sloppy reading)

We find this example to also be acceptable with the pair-list reading given in (53b), suggesting that it in fact receives a functional interpretation. The fact that it can also receive a singular functional NP as an answer as shown in (53c) provides further support for the functional analysis.

This example in fact satisfies all of the tests for functional readings that were failed by the examples we previously considered. For instance, as expected on a functional analysis, but in marked contrast to examples like (45a), a variant of (53a) with VP-coordination is unacceptable with a pair-list answer:

a. Which victim on his list did John maim on Tuesday and kill on Wednesday?

b. \# He maimed Bruno on Tuesday and killed Arno on Wednesday.

The same is true for a variant of the acceptable NP-coordination case (46), as shown in (55):

a. Which inhabitant of its coast did John kill in Africa and Asia?

b. \# He killed Bruno in Africa and Arno in Asia.

Thus, the evidence suggests that these cases are instances of functional readings.

In moving from Munn's (in our opinion, infelicitous) example (51) to his acceptable case in (52), two things changed: a pronoun (his) was added to the whphrase, and a nonrelational noun (man) was replaced with a relational one (victim). We therefore need to distinguish these in identifying the source of the difference in judgments. Example (56) is a variant of (53) with a relational noun but no pronoun:

(56) a. Which victim did John kill on Tuesday and Bill kill on Wednesday?

b. ?? John killed Bruno and Bill killed Arno.

c. ?? His brother. 
We find the answers in (56b-c) to be highly marginal, although perhaps not completely out. Example (51), which involves a non-relational noun, is still worse according to our judgments. As such, the ability to obtain functional readings with singular NPs appears to be gradient: Cases with NPs that combine a relational noun with a pronoun (53) are better than examples with a relational noun but no pronoun (56), which are in turn better than cases with only a nonrelational noun (51).

The difference between examples (53) and (56) is reminiscent of a difference that Sharvit (1999) cites with respect to functional readings for relative clauses. She notes that "even English headed relatives allow this [functional] reading, provided that the 'head' contains an anaphor" (p. 449), citing example (57a).

a. The picture of himself which every student hated annoyed his friends.

b. The picture which every student hated annoyed his friends.

c. The picture of his mother which every student hated annoyed his friends.

Sentence (57a) has a reading in which each student hated a picture of himself, for instance, his own passport photo. This reading appears to be absent (or certainly much less accessible) in example (57b), which is similar to (57a) but lacks the anaphor. Although Sharvit attributes the availability of a functional reading to the existence of an anaphor, we find that examples like (57c) are acceptable on a functional interpretation (e.g., every student hated his own mother's driver's license photo), in which a possessive pronoun is used on analogy with examples like (53a).

Regardless of whether one finds a functional reading for examples like (51) to be completely unacceptable or merely strongly dispreferred, the important fact for our purposes is that the plural examples we have considered - including Munn's cases that involve wh-NPs that are unspecified for number, e.g., Who, What, and Where - are cases of respective readings, and not functional readings. Pair-list responses for these examples (ex. 41) are fully felicitous, which patterns with analogous cases that involve unambiguously plural wh-NPs (ex. 44), but which puts them in marked contrast to analogous cases involving singular wh-NPs (ex. 43). Furthermore, these cases are not restricted in the way that examples with functional readings are; they are acceptable in examples with VP-coordination (ex. 45) and NP-coordination (ex. 46), for instance. Finally, the availability of respective interpretations in these examples is exactly what we would expect given the availability of such readings in a number of other filler-gap constructions, as discussed in Section 3. Indeed, it would have been odd if respective interpretations were not available in this particular extraction construction in light of these other data.

Before concluding, we briefly consider one more example, shown in (58), that at first blush might appear to involve a functional interpretation.

a. Which of their relatives did John and Bill visit?

b. John visited his aunt, and Bill visited his brother.

We are interested in the reading in which John and Bill have disjoint sets of relatives which they visited separately, per the pair-list answer given in (58b). This example 
bears a superficial resemblance to the functional reading examples we have considered such as (52a), although the syntactic configuration of (58a) does not allow for a Munn-style functional analysis. Indeed, the fact that the possessive pronoun their in (58a) is plural is a clue that the relevant reading is not functional.

Instead, the proper analysis of this example relies on our previous analysis of the adjective respective (Gawron and Kehler 2002), in which a RESP operator that takes NP-level scope enters the semantic composition, as is required to handle the analogous reading of example (59).

John and Bill visited their (respective) relatives.

Example (59) is consistent with the reading of interest whether or not the adjective respective is overt. (The example has other possible readings if respective does not appear, of course.) Example (58a) displays the same pattern; indeed the relevant reading is forced by the inclusion of respective:

(60) a. Which of their respective relatives did John and Bill visit?

b. John visited his aunt, and Bill visited his brother.

See Gawron and Kehler (2002) for further details of the analysis of examples like (59). Since RESP operators require plurals, the analysis correctly predicts that a variant of (58a) in which a singular NP is used, such as (61), does not admit of a respective (nor functional) interpretation:

Which relative of theirs did John and Bill visit?

The difference in available readings for (58a) and (61) contrasts with the lack thereof for their respective variants (52) and (53), the latter of which are both properly analyzed as functional, and not respective, readings.

\section{Conclusion}

To conclude, with one subclass of exceptions, Munn's examples are instances of respective readings and not functional readings. Our analysis captures these readings, as well as those for a range of other filler-gap constructions, since RESP operators routinely intervene between constituent-based dependencies in the syntax and predicate-argument relations in the semantics. As a result we are able to account for cases that share essential characteristics with Munn's examples but which are not candidates for a functional analysis. These same examples conspire to demonstrate that the identity constraint on ATB extraction cannot be maintained.

It is worth reiterating that none of the machinery that we have used here is motivated specifically by the need to handle such examples. Instead, this machinery is independently necessary to simultaneously capture a variety of adequacy criteria 
for any analysis of respective readings. These criteria include examples in which a respective reading applies over one or more groups that are not specified with a coordinate structure, cases of respective readings without the occurrence of the word respectively, and examples in which the respective reading applies over one or more groups that denote properties.

\section{Acknowledgments}

We thank Chris Barker for comments on a dry run of the talk we presented at the conference and two anonymous reviewers for comments on our abstract.

\section{Endnotes}

1. We and Munn disagree about the acceptability of this and other key examples, a point to which we return later.

2. A brief proof is provided in Gawron and Kehler (in press).

3. The (phonetically unrealized) DIST operator is included in the tree merely to indicate where it intervenes in the semantic composition, and not to suggest that it is somehow manifest in the syntax.

4. The approach we have taken here associates the RESP operator with the question, but it is also possible to associate it with the answer. We do not take a definitive stance on this question here, leaving it for further research.

5. Munn (1998), which came to our attention quite recently, briefly considers the possibility of a respective analysis, but rejects it on the basis of examples like (51). Munn (1999), which does not cite Munn (1998), makes no mention of this alternative.

\section{References}

Chierchia, Gennaro. 1993. Questions with quantifiers. Natural Language Semantics, 1:181-234.

Dalrymple, Mary and Andrew Kehler. 1995. On the constraints imposed by respectively. Linguistic Inquiry (Squibs and Discussion), 26(3):531-536.

Gawron, Mark and Andrew Kehler. 2002. The semantics of the adjective respective. In Proceedings of the 21st West Coast Conference on Formal Linguistics (WCCFL XXI), pages 85-98, Santa Cruz, CA, April.

Gawron, Mark and Andrew Kehler. In press. The semantics of respective readings, conjunction, and filler-gap dependencies. Linguistics and Philosophy.

Gazdar, Gerald. 1980. A cross-categorial semantics for conjunction. Linguistics and Philosophy, 3:407-409.

Krifka, Manfred. 2001. Quantifying into question acts. Natural Language Semantics, 9:1-40. 
Lahiri, Utpal. 2000. Lexical selection and quantificational variability in embedded interrogatives. Linguistics and Philosophy, 23:325-389.

Link, Godehard. 1983. The logical analysis of plurals and mass terms: A latticetheoretical approach. In Rainer Bauerle, Christoph Schwarze, and Arnim von Stechow, editors, Meaning, Use, and the Interpretation of Language. de Gruyter, Berlin, pages 302-323.

Massey, Gerald. 1976. Tom, Dick and Harry, and all the King's Men. American Philosophical Quarterly, 13:89-107.

McCawley, James D. 1998. The Syntactic Phenomena of English. University of Chicago Press, Chicago, second edition.

Munn, Alan. 1998. ATB movement without identity. In Jennifer Austin and Aaron Lawson, editors, Proceedings of the the 14th Eastern States Conference on Linguistics (ESCOL-97), pages 150-160. CLC Publications.

Munn, Alan. 1999. On the identity requirement of ATB extraction. Natural Language Semantics, 7:421-425.

Partee, Barbara and Mats Rooth. 1983. Generalized conjunction and typeambiguity. In Rainer Bauerle, Christoph Schwarze, and Arnim von Stechow, editors, Meaning, Use, and the Interpretation of Language. de Gruyter, Berlin, pages 361-383.

Roberts, Craige. 1987. Modal Subordination, Anaphora, and Distributivity. Ph.D. thesis, University of Massachusetts at Amherst.

Schwarzschild, Roger. 1996. Pluralities. Kluwer, Dordrecht.

Sharvit, Yael. 1999. Functional relative clauses. Linguistics and Philosophy, 22:447-478.

Winter, Yoad. 1996. A unified treatment of singular NP coordination. Linguistics and Philosophy, 19:337-391. 